\title{
Inoculación de Hordeun vulgare var Armida (cebada) con Burkholderia cepacia y Gluconacetobacter diazotrophicus
}

\author{
Inoculation of Hordeum vulgare var. Armida (barley) with Burkholderia cepacia and \\ Gluconacetobacer diazotrophicus
}

\section{Liliana Marquez-Benavidez ${ }^{1}$, Pedro Gabriel Morales ${ }^{1}$, Ivan Balderas-León ${ }^{1}$, Javier Villegas Moreno $^{1}$ y Juan Manuel Sánchez-Yáñez ${ }^{1^{*}}$}

\author{
${ }^{1}$ Microbiología Ambiental, Instituto de Investigaciones Químico Biológicas, Universidad Michoacana de San Nicolás \\ de Hidalgo, Ciudad Universitaria, México. \\ *Autor para correspondencia (syanez@umich.mx) \\ Recibido: 13/09/2015; Aceptado: 16/11/2015. \\ 10.18004/investig.agrar.2016.diciembre.87-94
}

\begin{abstract}
RESUMEN
La producción de cebada demanda la provisión adecuada de nitrógeno $(\mathrm{N})$ aplicado como fertilizante nitrogenado $(\mathrm{FN})$, que en exceso al igual que otras prácticas culturales inadecuadas podría causar pérdida de la productividad del suelo. Una alternativa para regular y optimizar el uso del FN en la cebada es la inoculación con géneros de bacterias promotoras del crecimiento vegetal endófitas (BPCVE), como Burkholderia cepacia y Gluconacetobacter diazotrophicus. El objetivo de este trabajo fue analizar el efecto de B. cepacia y $G$. diazotrophicus en cebada. El experimento se realizó en un invernadero bajo un diseño experimental factorial de seis tratamientos y seis repeticiones cada uno: a) cebada sin inocular y sin FN, o control absoluto (CA); b) sin inocular y $50 \%$ del FN, o control relativo 1 (CR1); c) sin inocular y $100 \%$ del FN, o control relativo 2 (CR2), d) inoculada con B. cepacia y $50 \%$ del $\mathrm{FN}$; e) inoculada con $G$. diazotrophicus y $50 \%$ del $\mathrm{FN}$ y f) inoculada con la mezcla de B. cepacia y G. diazotrophicus y el $50 \%$ del FN. Las variables analizadas fueron respuestas basadas en su fenología y biomasa: peso seco total (PST) a plántula y floración. Los resultados indicaron un efecto positivo de $B$. cepacia con $0,85 \mathrm{~g}$ de PST, valor sin diferencia estadística en comparación con los 0,54 g de PST de la cebada, control relativo (CR) con $\mathrm{FN}$ al $100 \%$ sin inocular. A floración B. cepacia ejerció un efecto positivo con 4,48 g de PST, al igual que la doble inoculación de $B$. cepacia y $G$. diazotrophicus con $3,29 \mathrm{~g}$ de PST. Estos valores tuvieron diferencia estadística en comparación a los $1,49 \mathrm{~g}$ de PST. Lo anterior sugiere que entre B. cepacia y la cebada existe una interacción específica, que favorece la mejor absorción radical del $\mathrm{FN}$ al $50 \%$, sin causar un problema de deficiencia nutricional en el crecimiento de la planta.
\end{abstract}

Palabras clave: colonización, absorción radical, bacterias promotoras del crecimiento endófitas.

\begin{abstract}
Cropping barley demands an adequate supply of nitrogen $(\mathrm{N})$ which can be applied as nitrogen fertilizer (NF) but if it is applied in excess, as any other inadequate cultural practice, it could lead to the loss of soil productivity. An alternative to reduce and optimize the use of NF in barley is its inoculation with genera of entophytic growth-promoting bacteria (EGPB), such as Burkholderia cepacia and Gluconacetobacter diazotrophicus. The aim of this research was to analyze the effect of B. cepacia and G. diazotrophicus on barley. The experiment was carried out under greenhouse conditions, with a unifactorial experimental design with 6 treatments and 6 replicates in each one: a) noninoculated barley and without NF (absolute control, AC); b) noninculated barley and $50 \%$ of $\mathrm{NF}$ (control 1, RC1); c) noninculated barley and $100 \%$ of $\mathrm{NF}$ (relative control 2,RC2), d) barley inoculated with B. cepacia and 50\% of NF; e) barley inoculated with $G$. diazotrophicus and $50 \%$ of NF and f) barley coinoculated with $B$. cepacia and $G$. diazotrophicus and $50 \%$ of NF. The analyzed variables were responses based on its phenotyping and biomass: total dry weight (TDW), at both seedlings and flowering stages. Results showed a positive response of $B$. cepacia with TDW of $0.85 \mathrm{~g}$ a value with no statistical difference when compared to the 0.54 gr TDW of noninculated barley and $100 \%$ of NF used as a relative control (RC). At flowering stage B. cepacia exerted a positive effect with a TDW of 4.48 g., which is equal to the effect caused with the coinculation of B. cepacia and $G$. diazotrophicus which produced a TDW of $3.29 \mathrm{~g}$. These values had a significant statistical difference when compared to the 1.49 grs of TDW These results suggest that there exists a specific interaction between B. cepacia and barley, which favors the best radical absorption of $50 \% \mathrm{NF}$ without causing any nutritional deficiency problem in plant growth.
\end{abstract}

Key words: colonization, radical absorption, endophytic plant growth-promoting bacteria. 


\section{INTRODUCCIÓN}

El rendimiento de Hordeum vulgare (cebada) disminuye por pérdida de fertilidad del suelo (Contreras-López et al. 2008); consecuencia en parte por prácticas culturales inadecuadas como riego superficial y sobreexplotación, así como el uso excesivo de fertilizante nitrogenado (FN), como $\mathrm{NH}_{4} \mathrm{NO}_{3}$ (nitrato de amonio) empleado en su cultivo, lo que provoca pérdida de la materia orgánica y contaminación ambiental (Palafox-Caballero et al. 2005). Una opción para regular y optimizar el uso de FN es la inoculación de las semillas de cebada con bacterias promotoras del crecimiento vegetal endófitas (BPCVE), que al colonizar el interior de sus raíces y el sistema de conducción vegetal, estimulan la formación de raíces primarias y secundarias adecuada para su crecimiento, y facilitan la absorción del FN (James 2000).

Las BPCVE tienen mayor disponibilidad de nutrientes en el interior de los tejidos vegetales y mínima competencia con otros géneros bacterianos relacionados, tal como sucede en la rizósfera y suelo (James 2000; Muthukumarasamy et al. 2002). Tal es el caso de los géneros de BPCVE Burkholderia cepacia y Gluconacetobacter diazotrophicus que invaden el sistema de conducción y en el interior del sistema radical, en donde los carbohidratos y ácidos orgánicos, metabolitos generados por la fotosíntesis les sirven como fuente de alimento; cuando colonizan y circulan en el xilema de una amplia variedad de plantas domésticas y silvestres (Sevilla et al. 2001; Youssef et al. 2004; Muñoz-Rojas et al. 2005). Las BPCVE pueden transformar esos metabolitos de la fotosíntesis en sustancias promotoras del crecimiento vegetal (SPCV) (Triplett 2000; Riggs et al. 2001), con las que ejercen efectos positivos en el crecimiento de gramíneas como la cebada.

Las BPCVE tienen una quimiotaxis positiva por los exudados orgánicos del sistema radical de la cebada, que para su conversión en SPCV en la proliferación de pelos radicales, los que en consecuencia aumentan el área de exploración y absorción del FN (Rojas et al. 2009), principalmente cuando su concentración se reduce. Como lo observado en otras Poaceas, al respecto Plana et al. (1999), reportaron la respuesta positiva de Triticum aestivum a B. cepacia y Azospirillum brasilense en función de su peso seco total (PST) con aumento del 20\%, en relación con el mismo PST de T. aestivum con la dosis del FN recomendada y sin tratar con esas BPCVE. Mientras que García-González et al. (2005), reportaron un incremento entre el 15 al $25 \%$ del peso fresco total (PFT) y PST de T. aestivum inoculado con Azospirillum spp. y
Azotobacter beijerinckii a dosis 50\% del FN respecto a la misma variable en $T$. aestivum usado como CR a la dosis del FN sugerido en la región, sin las BPCVE. Igualmente, Mora y Toro (2007) evaluaron el efecto positivo de Burkholderia sp. endófita en Zea mays mediante la síntesis de SPCV que indujeron un incremento en la longitud radical y biomasa entre el 28 y $52 \%$ respectivamente, en comparación con Z. mays empleado como CR a la dosis de FN recomendada en la región. A pesar de lo anterior, en la literatura no se reporta el uso de B. cepacia ni $G$. diazotrophicus, géneros de BPCVE en cebada a dosis $50 \%$ del FN.

El objetivo de este trabajo fue analizar la respuesta de cebada a la inoculación con Burkholderia cepacia y Gluconacetobacer diazotrophicus.

\section{MATERIALES Y MÉTODOS}

Se utilizó un suelo ubicado a los $19^{\circ} 39^{\prime}$ '27' de latitud norte y $100^{\circ} 19^{\prime} 59^{\prime \prime}$ 'de longitud oeste, con una altitud de $1.820 \mathrm{msnm}$ clima templado, temperatura media anual de $17,3^{\circ} \mathrm{C}$, precipitación anual de $796 \mathrm{~mm}$, granizadas promedio 3-4/año, heladas 4-8/año e insolación 227:63 h:min; en un terreno agrícola denominado "La cajita" de la Tenencia Zapata del municipio de Morelia, Mich., sobre el km 5 de la carretera Morelia- Pátzcuaro, México.

El suelo fue solarizado para minimizar el problema de plagas y enfermedades (Sánchez-Yáñez 2007). El área cuenta con un historial agrícola de 20 años con sistema de cultivo intensivo cereal-cereal: maíz-trigo y maíz-cebada. El suelo fue tipificado como lateritico sódico según la clasificación de la FAO (2007): de textura arcillosa, con bajo contenido de materia orgánica $\left(15 \mathrm{~g} \mathrm{~kg}^{-1}\right)$, y de $\mathrm{N}$ orgánico (39 $\left.\mathrm{kg} \mathrm{ha}{ }^{-1}\right), \mathrm{pH} \quad 6,7$ ligeramente ácido (Whitmore 2000).

El género y especie de $B$. cepacia se aisló de las raíces de $Z$. mays sp. mexicana (teocintle) en un medio de cultivo conocido como agar Pseudomonas cepacia ácido azelaico y triptamina (APCAT) (Tran Van et al. 2000). Este aislado se identificó mediante su perfil bioquímico con el sistema API-50CH (BioMerieux, Francia).

Según se describe en la literatura, B. cepacia endófita de $Z$. mays sp. mexicana, se comparó con las siguientes cepas de referencia: B. cepacia ATCC 2546 y $B$. vietnamiensis TVV75. En relación al aislado de $G$. diazotrophicus se recuperó de Saccharum officinarum en caldo y Agar Lisina Glucosa Indol (ALGI) incubado a $32^{\circ} \mathrm{C}$ por $24 \mathrm{~h}$ (Youssef et al. 2004; Dibut et al. 2005); este aislado se identificó a nivel de especie con el sistema 
API20NE (Salazar et al. 2008). Para demostrar que $B$. cepacia y $G$. diazotrophicus tenían la capacidad de sintetizar SPCV, en específico algún tipo de giberelina, cada uno se sembró en APCAT y AGLI. Para ello se suprimió el agar y en medio líquido se cultivó cada género de BPCVE en matraces de $250 \mathrm{~mL}$ con $100 \mathrm{~mL}$ APCT y AGLI. Los matraces se agitaron a $250 \mathrm{rpm}$ con un mínimo de $24 \mathrm{~h}$ y un máximo de $48 \mathrm{~h}$, temperatura $30^{\circ} \mathrm{C}$ en función del crecimiento suficiente para iniciar la inoculación de la semilla de cebada. Posteriormente cada matraz se centrifugó a $3.000 \mathrm{rpm} / 15 \mathrm{~min}$; luego se eliminó el paquete celular y el sobrenadante se filtró con una membrana milipore $0,2 \mu$ estéril (Gelman, Co); para obtener el filtrado estéril libre de células de B. cepacia y $G$. diazotrophicus: que contenían las SPCV relacionadas con algún de tipo giberelina.

Como referencia positiva para la germinación de las semillas se aplicaron 25 ppm de giberelina (Sigma, Co) pura disuelta en agua estéril y como control absoluto (CA); agua estéril. Con base en los siguientes tratamientos: a) los filtrados estériles libres de células de B. cepacia y G. diazotrophicus; b) giberelina pura; c) agua estéril. Se emplearon 100 semillas de $H$. vulgare, $P$. vulgaris y $Z$. mays respectivamente; colocadas en caja de Petri sobre algodón humedecido esterilizado, mantenidas a temperatura entre $25-30^{\circ} \mathrm{C} / 9$ días. Para este ensayo se midieron las variables respuesta: porcentaje y días de germinación (Singh et al. 2003; García-González et al. 2005; Tsavkelova et al. 2006); Burkholderia cepacia y Gluconacetobacter diazotrophicus fueron inoculados en la semilla de cebada, para lo que por cada 200 semillas se les aplicó 1,0 mL de B. cepacia o G. diazotrophicus a una densidad de $600 \times 10^{6}$ UFC $\mathrm{mL}^{-1}$ calculado con base en una cuenta viable en placa en APCAT y ALGI respectivamente. Cuando se realizó la doble inoculación con B. cepacia y G. diazotrophicus por cada 200 semillas de cebada se usó $1,0 \mathrm{~mL}^{-1}(0,5 \mathrm{~mL}$ de cada una) en una proporción 1:1 (Bashan y Levanony 1998; GarcíaGonzález et al. 2005).

En la Tabla 1 se muestran los tratamientos utilizados para medir el efecto de las BPCVE en $H$. vulgare var. Armida a la dosis $50 \%$ del FN como $\mathrm{NH}_{4} \mathrm{NO}_{3} 5 \mathrm{~g} \mathrm{~L}^{-1}$. En Michoacán, México para la cebada se recomienda una dosis de $\mathrm{FN}$ equivalente a $10 \mathrm{~g} \mathrm{~L}^{-1}$ de $\mathrm{NH}_{4} \mathrm{NO}_{3}$ (Cortés y Ortiz 2008; Villaseñor et al. 2014). Este ensayo se realizó en invernadero, ahí el suelo se colocó en el sistema hidropónico de jarras de Leonard (García-González et al. 2005); en el reservorio de cada jarra de Leonard se agregó la $\mathrm{SM}$ con la siguiente composición química $\left(\mathrm{g} \mathrm{L}^{-1}\right)$ : $\mathrm{NH}_{4} \mathrm{NO}_{3} 10,0 ; \mathrm{K}_{2} \mathrm{HPO}_{4} 2,5 ; \mathrm{KH}_{2} \mathrm{PO}_{4} 2,0 ; \mathrm{MgSO}_{4}$ 1,0; $\mathrm{NaCl} 0,1 ; \mathrm{CaCl}_{2} 0,1 ; \mathrm{FeSO}_{4}$ trazas, ajustado a $\mathrm{pH} 6,5$ y $10,0 \mathrm{~mL}$ de solución de oligoelementos con la siguiente composición (g L $\left.{ }^{-1}\right): \mathrm{H}_{3} \mathrm{BO}_{3}$ 2,86, $\mathrm{ZnSO}_{4} 7 \mathrm{H}_{2} \mathrm{O} \quad 0,22$ : $\mathrm{MnCl}_{2} 7 \mathrm{H}_{2} \mathrm{O} 1,81 ; \mathrm{K}_{2} \mathrm{MnO}_{4} 0,09$; ajustado a pH 6,8; se usaron $500 \mathrm{~mL} / 15$ días/jarra de Leonard. En el suelo de cada jarra de Leonard, la humedad se mantuvo al $80 \%$ de capacidad de campo, con agua potable por 14 semanas (Sánchez-Yáñez 2007).

Los tratamientos fueron dispuestos bajo un diseño experimental unifactorial de seis tratamientos y seis repeticiones en cada uno: a) cebada sin inocular, sin FN (control absoluto, CA); b) cebada sin inocular con el 50\% de FN (control relativo 1, CR1); c) cebada sin inocular con el $100 \%$ de FN (control relativo 2, CR2), d) cebada inoculada con B. cepacia y el $50 \%$ de $\mathrm{FN}$; e) cebada inoculada con $G$. diazotrophicus y $50 \%$ del FN y f) cebada inoculada con la mezcla de B. cepacia y $G$. diazotrophicus y el 50\% del FN (García-González et al. 2005).

En la Tabla 1 se muestra el diseño experimental empleado para evaluar la respuesta de la cebada a la inoculación con B. cepacia y G. diazotrophicus a nivel de plántula y de floración, 32 y 62 días después de la siembra. Las variables respuesta evaluadas fueron: altura de la planta (AP) y longitud de la raíz (LR), ambas medidas tomadas con una regla calibrada. Para la AP se consideró desde la base del cuello hasta el ápice máximo de crecimiento; para la LR se tomó desde el cuello de la planta a la longitud máxima de la raíz principal. También fueron registrados los valores para biomasa: peso fresco total (PFT), para ello se tomó una planta recién colectada, se seleccionó la parte aérea que se pesó en una balanza electrónica, y para el peso seco total (PST) la planta se secó en un horno a $75^{\circ} \mathrm{C} / 30 \mathrm{~h}$ hasta registrar un peso constante.

Los datos experimentales se analizaron por ANOVA y Tukey con un nivel de significancia de $\mathrm{P}>0,05$ para establecer las diferencias en los resultados obtenidos, cuando la cebada fue inoculada con B. cepacia y/o $G$. diazotrophicus a la dosis $50 \%$ del FN, en comparación con la cebada sin inocular con el FN al $100 \%$ (García et al. 2001). 
Tabla 1. Tratamientos para evaluar el efecto de la inoculación de Burkholderia cepacia y Gluconacetobacter diazotrophicus en Hordeum vulgare var. Armida.

Tratamiento

1. Cebada irrigada solo con agua (control absoluto)

2. Cebada alimentada con solución mineral 50\%, sin inocular con B. cepacia y/o G. diazotrophicus (control relativo 1)

3. Cebada alimentada con solución mineral $100 \%$ sin inocular con B. cepacia y/o G. diazotrophicus, (control relativo 2 )

4. Cebada alimentada con solución mineral $50 \%$, inoculada con

Burkholderia cepacia

5. Cebada alimentada con solución mineral $50 \%$, inoculada con

Gluconacetobacter diazotrophicus

6. Cebada alimentada con solución mineral 50\%, inoculada con B. cepacia y

G. diazotrophicus
Fertilizante nitrogenado (FN) $\mathrm{NH}_{4} \mathrm{NO}_{3}$

Se aplicó (+), No se aplicó (-)

\section{RESULTADOS Y DISCUSIÓN}

Efecto de Burkholderia cepacia y Gluconacetobacter diazotrophicus fue observado en la cebada con el FN al $50 \%$ (Figura 1), en las fases de plántula y floración; donde se verificó que $B$. cepacia registró un valor de $43,75 \mathrm{~cm}$ de la AP, sin diferencia estadística con los 41,0 cm de la cebada con la dosis $100 \%$ de FN o CR; pero sí con los 31,12 cm de AP de la cebada irrigada con agua o CA. Esto sugiere que el efecto positivo de B. cepacia en el interior del tejido de raíz de la cebada, fue el resultado de la transformación de algunos de los compuestos orgánicos derivados de la fotosíntesis en SPCV para su sano crecimiento (García et al. 2001).

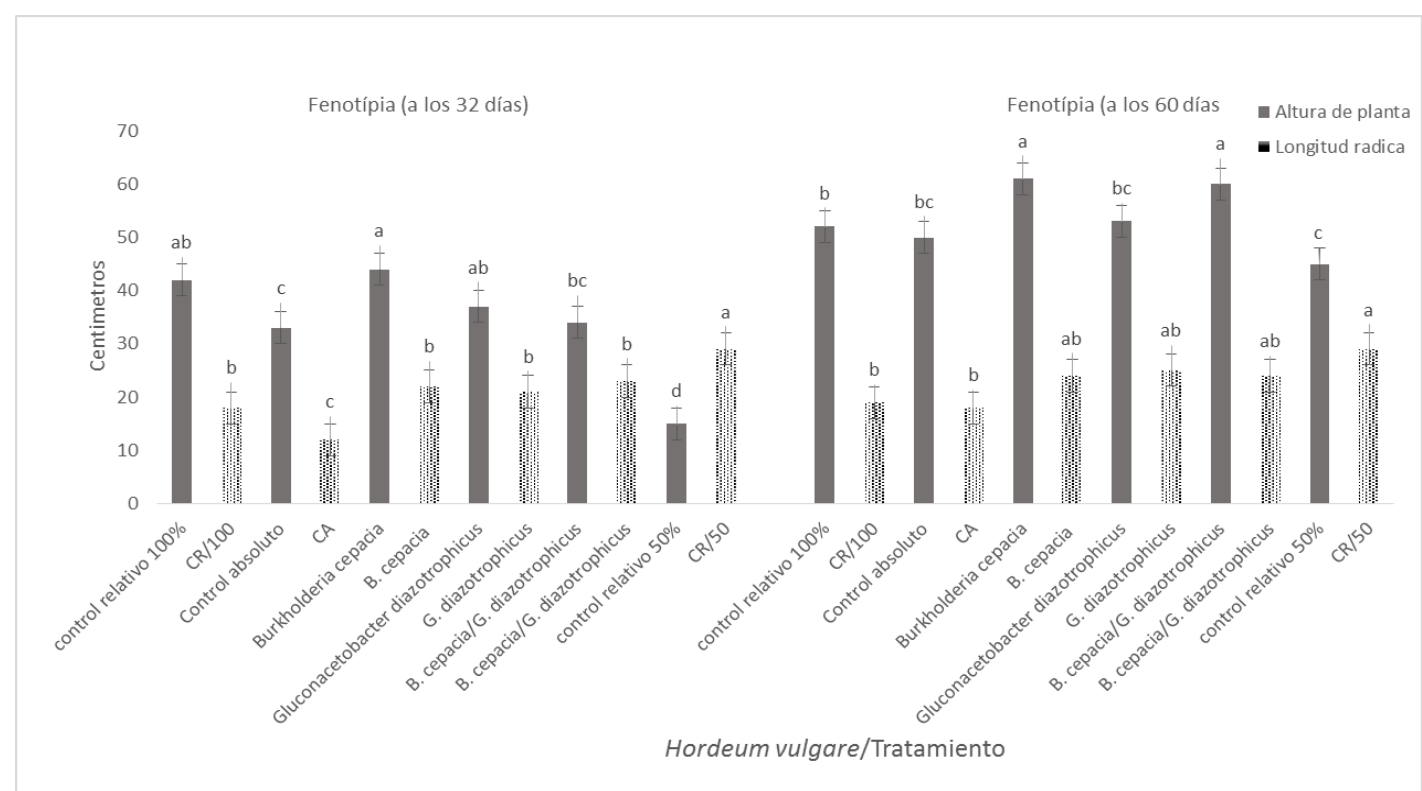

Valores de medias \pm error estándar, seguidas por letras distintas con diferencia estadística significativa (ANOVA, P>0,05, Tukey HSD).

Figura 1. Efecto de la inoculación de Burkholderia cepacia y Gluconacetobacter diazotrophicus en Hordeum vulgare var. Armida en la fenología a plántula y floración. 
La respuesta positiva de la cebada a $B$. cepacia y $G$. diazotrophicus, en relación a LR, registraron un valor de $23,5 \mathrm{~cm}$, sin diferencia estadística con los $18,5 \mathrm{~cm}$ con la LR de la cebada alimentada con la dosis $100 \%$ del FN o CR. Sin embargo, ambos valores fueron estadísticamente diferentes e inferiores a los 12,0 cm de la cebada irrigada solo con agua o CA. Este resultado indica que los dos géneros de BPCVE mediante SPCV, indujeron una mayor proliferación de raíces secundarias que incrementó su capacidad de absorción radical del FN, a pesar de reducir la dosis al 50\%. Este fue un resultado positivo, análogo a lo reportado en $Z$. mays inoculado con la endófita Burkholderia sp. con la dosis del FN inferior a lo recomendado para esa gramínea (Riggs et al. 2001; Mora y Toro 2007).

Se observó un efecto positivo de B. cepacia en la cebada a floración con una AP de $61,0 \mathrm{~cm}$, así como con los 58,62 $\mathrm{cm}$ de la cebada tratada con $B$. cepacia y $G$. diazotrophicus, ambos valores fueron estadísticamente diferentes y superiores en comparación con los $52,0 \mathrm{~cm}$ registrados en el tratamiento CR y con los 49,0 cm del CA. Estos resultados sugieren que en el xilema de la planta, B. cepacia como G. diazotrophicus actuaron simultáneamente en la generación de SPCV, y con ello para una eficaz absorción radical del $\mathrm{FN}$ a pesar de reducirlo al 50\%. También se observó un efecto benéfico de $G$. diazotrophicus en la LR de la cebada, con 25,33 $\mathrm{cm}$; al igual que con los $24,56 \mathrm{~cm}$ de la planta tratada con B. cepacia, ambos valores fueron estadísticamente diferentes y superiores respecto a los 19,3 cm de la LR obtenida en el CR y los $18,5 \mathrm{~cm}$ del tratamiento solo con agua o CA. Estos resultados sugieren que los dos géneros de BPCVE, al colonizar y proliferar en el tejido interno de la raíz de la cebada, convirtieron algunos de los aminoácidos derivados de la fotosíntesis en SPCV lo que optimizó el FN a pesar de reducirlo al 50\% (Chanway et al. 2000; Bent et al. 2001; Muthukarasamy et al. 2002; Rojas et al. 2009).

Efecto positivo de Burkholderia cepacia y Gluconacetobacter diazotrophicus en Hordeum vulgare var. Armida fue observado en la variable analizada PFT, en especial con B. cepacia, en la que se obtuvo 7,78 g, valor estadísticamente diferente y superior a los 3,52 g obtenido con la dosis del FN al $100 \%$ o CR y los $1,87 \mathrm{~g}$ con el tratamiento irrigado solo con agua o CA. Esto puede indicar que B. cepacia en el interior de la raíz de la cebada, convirtió algunos compuestos orgánicos derivado de la fotosíntesis en SPCV, causando un incremento de la absorción y optimización de la dosis del FN al 50\%, sin afectar su crecimiento (García et al. 2001). Además, $B$. cepacia en la cebada registró un PST de 0,85 g valor sin diferencia estadística en comparación con los 0,54 g del tratamiento con el $100 \%$ del FN o CR; estos valores fueron estadísticamente distintos a los $0,23 \mathrm{~g}$ del CA.

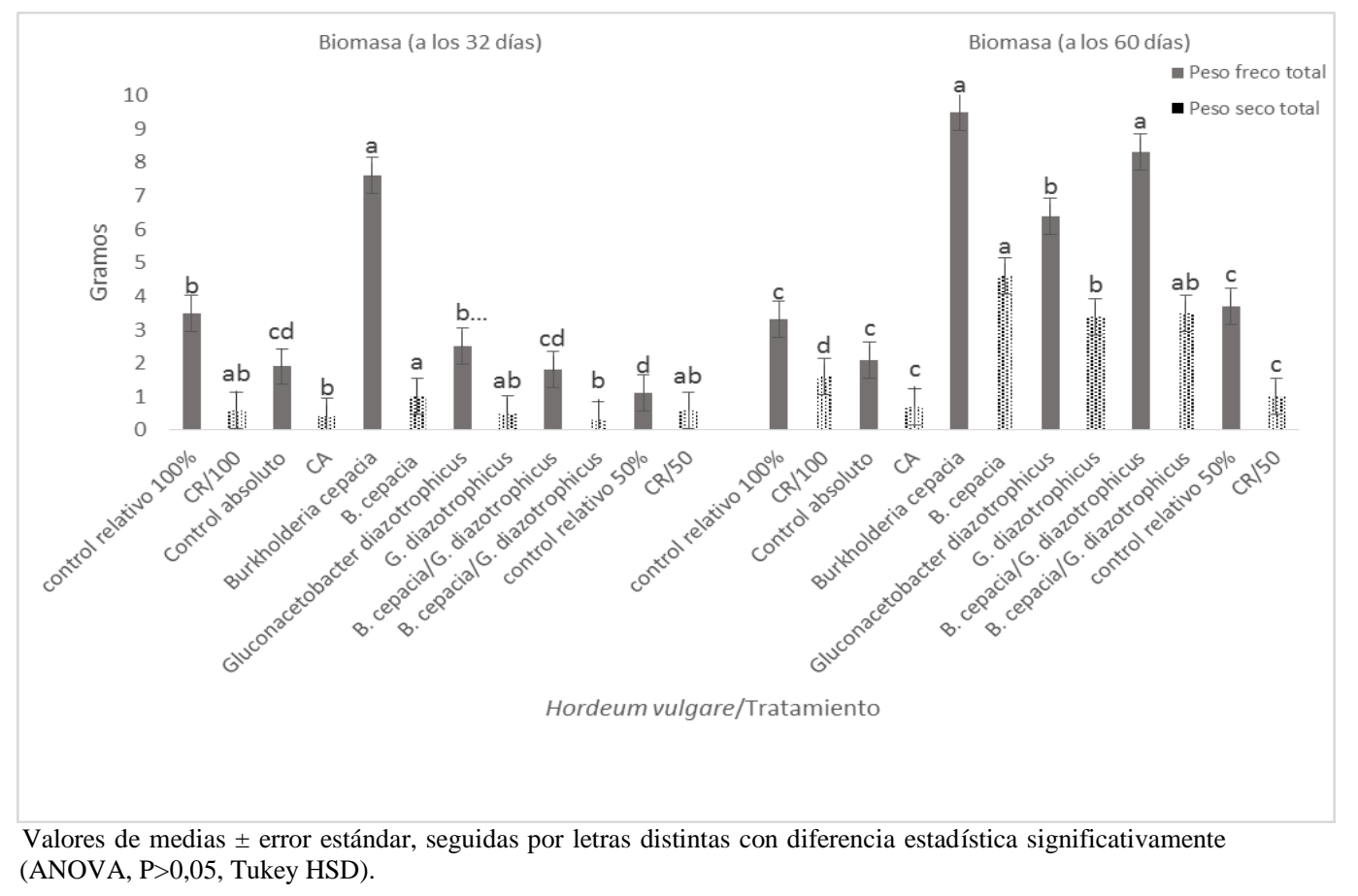

Figura 2. Efecto de la inoculación de Burkholderia cepacia y Gluconacetobacter diazotrophicus en Hordeum vulgare var. Armida en su biomasa a plántula y floración. 
B. cepacia registró 9,62 g de PFT y G. diazotrophicus $8,22 \mathrm{~g}$, ambos valores sin diferencia estadística en comparación con el PFT de la cebada del CR, lo que sugiere que en el xilema de esta planta las BPCVE mediante la generación de SPCV mejoraron y optimizaron la dosis del FN al 50\% (Mora y Toro 2007). La inoculación de BPCVE en $Z$. mays indujo un incremento en la formación de raíces secundarias, con un aumento en la capacidad de absorción radical del FN al $50 \%$, sin que el resultado haya sido un crecimiento anormal del Z. mays, en comparación con el Z. mays tratado con el FN al $100 \%$ sin inocular con las BPCVE (Riggs et al. 2001; Mora y Toro 2007). Estos valores de PFT (9,62 y 8,22 g) fueron estadísticamente diferentes y superiores a los 3,22 g de PFT obtenido con la dosis del $\mathrm{FN}$ al $100 \%$ o CR y los 2,0 g del tratamiento irrigado con agua o $\mathrm{CA}$.

Cuando B. cepacia se inoculó en la cebada se registró 4,48 g de PST, mientras que con la mezcla de B. cepacia y G. diazotrophicus se obtuvo 3,29 g para la misma variable. Ambos valores fueron estadísticamente diferentes y superiores a los 1,49 g de PST obtenido en el CR y los 0,66 g del CA. Este hecho apoya que en el xilema de la cebada, tanto $B$. cepacia como $G$. diazotrophicus sintetizaron SPCV, que aumentaron su capacidad de absorción radical del FN al 50\% para su optimización y permitir su sano crecimiento (Chanway et al. 2000; Muthukarasamy et al. 2002). Dado que B.cepacia como G. diazotrophicus endófitos de plantas, tienen la capacidad genética de convertir metabolitos de la fotosíntesis en SPCV, con una acción similar a la de ciertas auxinas y giberelinas (Bent et al. 2001), específicamente cuando géneros y especies de BPCVE se asocian con las Poaceas del tipo $S$. officinarum y lo novedoso e interesante también con $H$. vulgare (Rojas et al. 2009) de acuerdo a los resultados obtenidos en este investigación.

\section{CONCLUSIONES}

Las BPCVE como B. cepacia causan un efecto positivo en el crecimiento de la cebada, no obstante disminuir la dosis del FN al 50\%, en especial en comparación a lo que se registra cuando se inocula con G. diazotrophicus.

El hecho de que ambos géneros de BPCVE colonicen el xilema de la planta, sugiere que la respuesta positiva de la cebada es relativamente posible cuando se inocula con $B$. cepacia, a pesar de reducir la dosis del FN al 50\%, inferior a lo recomendado de acuerdo a la variedad de cebada y tipo de suelo, sin afectar negativamente su crecimiento, lo que aunado a prácticas culturales de conservación agrícola evitan la perdida de la fertilidad del suelo.

\section{AGRADECIMIENTOS}

Al Dr. Tran Van del Departamento de Bioquímica y Microbiología del Suelo de la Universidad Claude Bernard, Lyon, Francia por la donación de las cepas $B$. cepacia ATCC 2546 y B. vietnamiensis TVV75.

Al proyecto 2,7 (2016) de la Coordinación de Investigación Científica de la UMSNH, Morelia, Mich, a BIONUTRA, SA de CV, Maravatio, Mich, México.

\section{REFERENCIAS BIBLIOGRAFICAS}

Bashan, Y; Levanony, H. 1998. Interaction between Azospirillum brasilense $\mathrm{Cd}$ and wheat root cells during early stages of root colonization. In: Klingmuller, W. (ed.). Azospirillum IV. Berlin, Germany, Springer-Verlag. p. 166-173.

Bent, E; Tuzun, S; Chanway, CP; Enebak, S. 2001. Alterations in plant growth and in root hormone levels of lodgepole pines inoculated with rhizobacteria. Canadian Journal of Microbiology 47:793-800.

Chanway, CP; Shishido, M; Nairn, J; Jungwirth, S; Markham, J; Xiao, G; Holl, FB. 2000. Endophytic colonization and field responses of hybrid spruce seedlings after inoculation with plant growthpromoting rhizobacteria. Forest Ecology and Management 133:81-88.

Contreras-López, E; Jaimez-Ordaz, J; HernándezMadrigal, T; Añorve-Morga, J; Beltrán-Hernández, R. 2008. Composición química de cebadas cultivadas bajo diferentes condiciones de labranza en tres localidades del estado de Hidalgo, México. Bioagro 20:201-208.

Cortés, JM; Ortiz, A. 2008. Recomendaciones de fertilización para el cultivo de trigo. In $\mathrm{V}$ jornada de transferencia de tecnología del cultivo de trigo (en línea). México, Fundación Produce Sinaloa. p. 7-18. Consultado 10 jun 2015. Disponible en: http://www.fps.org.mx/divulgacion/ attachments/article/1009/v-jornada-trigo.pdf

Dibut, B; Ortega, M; Martínez, R; Fey, L; Ríos, Y. 2005. Nuevos aislados de Gluconacetobacter diazotrophicus 
en cultivos de importancia económica para Cuba. Cultivos tropicales 26: 5-10.

FAO (Organización de las Naciones Unidas para la Alimentación y la Agricultura, Italia). 2007. Base referencial del recurso suelo: Un marco conceptual para clasificación, correlación y comunicación. Roma, Italia, FAO. Informes sobre Recursos Mundiales de Suelos No. 103.

García, JA; Castillo, A; Ramírez, ME.; Rendón, G; Larqué, MU. 2001. Comparación de los procedimientos de Tukey, Duncan, Dunnett, HSU y Bechhofer para selección de medias. Agrociencia 35:79-86.

García-González, MM; Farías-Rodríguez, R; PeñaCabriales, JJ; Sánchez-Yáñez, JM. 2005. Inoculación del trigo var. Pavón con Azospirillum spp. y Azotobacter beijerinckii. Terra Latinoamericana 23:65-72.

James, EK. 2000. Nitrogen fixation in endophytic and associative symbiosis. Field Crops Research 65:197209.

Mora, E. y Toro, M. 2007. Estimulación del crecimiento vegetal por Burkholderia cepacia, una cepa nativa de suelos ácidos de sabanas venezolanas. Agronomía Tropical 57:123-128.

Muñoz-Rojas, J; Fuentes-Ramirez, LE.; CaballeroMellado, J. 2005. Antagonism among Gluconacetobacter diazotrophicus strains in culture media and in endophytic association. FEMS Microbial Ecology 54:57-66.

Muthukumarasamy, R; Revathi, G; Seshadri, S; Lakshminarasimhan, C. 2002. Gluconacetobacter diazotrophicus (syn. Acetobacter diazotrophicus), a promising diazotrophic endophyte in tropics. Current Science 83:137-145.

Palafox-Caballero, A; Tosquy-Valle, O; Sierra-Macías, M; Turrent-Fernández, A; Espinosa-Calderón, A. 2005. Response of common and high quality protein corn hybrids to chemical fertilization. Terra Latinoamericana 23:129-135.

Plana, R; Medina, N; Moreno, I; Ramírez, A. 1999. Efecto agronómico de la biofertilizacion con dos rizobacterias en la producción de trigo (Triticum aestivum L.) en cuba. Cultivos tropicales 20:5-8.

Riggs, PJ; Chelius, MK; Iniguez, AL.; Kaeppler, SM; Triplett, EW. 2001. Enhanced maize productivity by inoculation with diazotrophic bacteria. Australian Journal of Plant Physiology 28:829-836.

Rojas, MM; Rodríguez, AJ; Trujillo, ID; Heydrich, M. 2009. Relación de la fijación de nitrógeno y la producción de auxinas en las cepas de Gluconacetobacter diazotrophicus procedentes de diferentes cultivos. Revista Colombiana de Biotecnología 6:84-93.

Salazar, EZ; Nieves, B; Ruíz, J; Vila, J. 2008. Utilidad del Sistema API 20NE para identificar especies del género Acinetobacter y otros bacilos Gram negativos no fermentadores. Sociedad Venezolana de Microbiología 28: 89-95.

Sánchez-Yáñez, JM. 2007. Breve tratado de microbiología agrícola: teoría y práctica. Morelia, México, Instituto de Investigaciones Químico Biológicas, Universidad Michoacana de San Nicolás de Hidalgo, COSUSTENTA, Centro de Investigación y Desarrollo del Estado de Michoacán p.130-133.

Sevilla, M; Burris, RH; Nirmala-Gunalapa, GL; Kennedy, C; Gunalapa, N. 2001. Comparison of benefit to sugarcane plant growth and $15 \mathrm{~N} 2$ incorporation following inoculation of sterile plants with Acetobacter diazotrophicus wild-type and Nif- Mutant strains. Molecular Plant-Microbe Interactions 14:358366.

Singh, UP; Sarma, BK; Singh, DP. 2003. Effect of plant growth-promoting rhizobacteria and culture filtrate of Sclerotium rolfsii on phenolic and salicylic acid contents in chickpea (Cicer arietinum). Current Microbiology. 46:131-140.

Tran Van; Berge, O; Ngoke, S; Balandreu, J; Heulin, T. 2000. Repeated beneficial effect of rice inoculation with a strain of Burkholderia vietnamiensis on early and late yield components in low fertility sulphate acid soils of Vietnam. Plant Soil 218:273-284.

Triplett, EW. 2000. Prokaryotic nitrogen fixation: a model system for the analysis of a biological process. 
Wymondham, England, Horizon Scientific Press. 800 p.

Tsavkelova, E; Klimova, S; Cherdyntseva, T; Netrusov, A. 2006. Microbial producers of plant growth stimulators and their practical use: a review. Applied Biochemestry Microbiology 42: 117-126.

Villaseñor, MH; Espitia, RE.; Huerta, EJ; Solas, ME; Ireta, MJ; Osorio, AL; Perez, HP. 2014. Nana F2007, cultivar de trigo para siembras de temporal en México. Revista Mexicana de Ciencias Agrícolas 7: 13631368.
Whitmore, AP. 2000. The biological management of soil fertility project. NJAS-Wageningen Journal of Life Sciences 48:115-122.

Youssef, H; Fayez, M; Monib, M; Hegazi, N. 2004. Gluconacetobacter diazotrophicus: a natural endophytic diazotroph of Nile Delta sugarcane capable of establishing an endophytic association with wheat. Biology and Fertility of Soils 39:391-397 